\title{
Tendências recentes da produção em história da enfermagem no Brasil ${ }^{*}$
}

\author{
Recent trends in scholarship on \\ the history of nursing in Brazil
}

\author{
Maria Itayra Padilha \\ Professora do Departamento de \\ Enfermagem e do Programa de \\ Pós-graduação em Enfermagem/ \\ Universidade Federal de Santa \\ Catarina (PEN/UFSC). \\ Rodovia Amaro Antônio Vieira, \\ $2371 / 818$ \\ 88034-102 - Florianópolis - SC - \\ Brasil \\ padilha@nfr.ufsc.br
}

Aline Coelho Ferreira

Mestranda do PEN/UFSC; enfermeira Hospital SOS

Cárdio-Florianópolis.

Rodovia João Gualberto Soares, 5284 88032-001 - Florianópolis - SC Brasil

alineferreiraufsc@yahoo.com.br

Isabel Cristina Alves

Maliska

Enfermeira do Hospital

Universitário/UFSC.

Rua Trajano Margarida, 144/bloco

$\mathrm{A} / 203$

88036-050 - Florianópolis - SC -

Brasil

isabel.alves07@yahoo.com.br

\section{Mariana Vieira \\ Villarinho}

Enfermeira/Secretaria de Estado da Administração.

Rodovia Virgílio Várzea, 2236/602C 88032-001 - Florianópolis - SC Brasil

nanyufsc@ibest.com.br

\section{Gabriela Venier \\ Zytkuewisz}

Enfermeira/Prefeitura Municipal de Florianópolis.

Rodovia SC 401

88000-000 - Florianópolis - SC -

Brasil

gabivenier@yahoo.com.br

\section{Camilla Sell}

Enfermeira/Secretaria de Estado da Saúde.

Rua Irmã Benwarda, 297

88015-270 - Florianópolis - SC -

Brasil

camillasell@hotmail.com
PADILHA, Maria Itayra et al. Tendências recentes da produção em história da enfermagem no Brasil. História, Ciências, Saúde - Manguinhos, Rio de Janeiro, v.20, n.2, abr.-jun. 2013, p.695-707.

\section{Resumo}

Visa analisar a produção científica dos grupos de pesquisa em história da enfermagem no Brasil de 1999 a 2009. A partir do estudo exploratório descritivo documental na versão 5.0 do diretório dos grupos de pesquisa do Conselho Nacional de Desenvolvimento Científico e Tecnológico (CNPq), e nos currículos individuais dos pesquisadores líderes disponibilizados na Plataforma Lattes, foram identificados 34 grupos de pesquisa com produção de 2.206 artigos, 230 livros, 745 capítulos de livros e 1.603 trabalhos completos e resumos expandidos. Há 394 artigos específicos de história da enfermagem nos temas identidade profissional, educação, institucionalização, especialidades e entidades organizativas. Conclui-se que a divulgação e socialização dos conhecimentos demonstram que essa área já está consolidada, mesmo que nem todos os artigos estejam diretamente relacionados à história da enfermagem.

Palavras-chave: grupos de pesquisa; história da enfermagem; história; produção científica

\section{Abstract}

The article analyzes the scholarship on the history of nursing in Brazil produced by research groups from 1999 to 2009. A descriptive and documental study of an exploratory nature was conducted, using version 5.0 of the National Council for Scientific and Technological Development's directory of research groups as well as the individual curricula of lead researchers, as available on the Lattes Platform. The result was the identification of 34 research groups, which produced 2,206 articles, 230 books, 745 book chapters, and 1,603 complete papers and expanded abstracts. The study found 394 specific articles on the history of nursing which explored the following topics: professional identity, education, institutionalization, specialties, and organizing bodies. It is concluded that the dissemination and socialization of this knowledge indicate that the area already has firm roots, even if all the articles were not directly related to the history of nursing.

Keywords: research groups; history of nursing; history; scientific production. 
$A_{\text {per }}$ história da enfermagem enquanto área de conhecimento reconhecida pelos seus pares e pelas demais áreas vem se consolidando no Brasil ao longo dos últimos 25 anos motivada, especialmente, por duas importantes questões. A primeira delas, em nossa avaliação, se deve à ampliação gradativa dos programas de pós-graduação em enfermagem, influenciando na produção de teses e dissertações com a perspectiva historiográfica. A segunda, como consequência da primeira, se deve à criação dos grupos de pesquisa voltados para a história da enfermagem.

O primeiro grupo de pesquisa com estudos na perspectiva histórica na área da enfermagem foi criado em 1988 e denominado Grupo de Pesquisa sobre Políticas e Práticas de Saúde (Grupps) da Universidade Federal do Ceará (UFC). Na década de 1990, foram criados nove grupos, e os outros 23 a partir de 2000, totalizando, atualmente, 34 grupos (Padilha et al., 2012).

A maioria dos grupos de pesquisa está vinculada aos programas de pós-graduação, que têm servido de mola propulsora para a produção do conhecimento em todas as áreas (Erdmann et al., 2005). A visibilidade do conhecimento produzido nesses programas e nos grupos de pesquisa se torna evidente a partir da publicação dos resultados dos estudos de pesquisadores, docentes e estudantes participantes. A pós-graduação stricto sensu está consolidada no cenário educacional brasileiro e internacional e tem contribuído decisivamente para a formação de recursos humanos qualificados e para o desenvolvimento científico-tecnológico nacional, deixando claro o seu papel estratégico no país (Padilha et al., 2012; Brasil, 2011a).

Na enfermagem brasileira, a pós-graduação stricto sensu teve início em 1972 com a criação do mestrado em enfermagem fundamental, na Escola de Enfermagem Anna Nery, da Universidade Federal do Rio de Janeiro (UFRJ). No ano seguinte, a Escola de Enfermagem da Universidade de São Paulo (Eeusp), iniciou o mestrado na área de concentração em fundamentos de enfermagem (Brasil, 2009, 2011b). Nota-se que a pós-graduação na enfermagem brasileira, em especial a oferta de doutores em enfermagem, acompanhou as demais áreas, crescendo significativamente nos últimos anos, atingindo visibilidade no sistema de ensino superior do país. No entanto, esse crescimento ocorre de forma desigual nas diferentes regiões do Brasil, e essa desigualdade também se reflete nas iniciativas de atividades de pesquisa em parceria com investigadores internacionais. Elas existem, porém ainda são tímidas e limitadas a alguns programas de pós-graduação fortemente consolidados, principalmente na região Sudeste do país (Rodrigues et al., 2002). De acordo com dados da Coordenação de Aperfeiçoamento de Pessoal de Nível Superior (Capes), atualmente há no Brasil cinquenta programas de pós-graduação stricto sensu em enfermagem. Os programas estão distribuídos da seguinte forma: 24 na região Sudeste; três na região Centro-Oeste; nove na região Nordeste; dez na região Sul; e quatro na região Norte. Considerando que, em 1998, tínhamos 14 programas, verificamos que houve um crescimento de $350 \%$ ao longo de apenas 12 anos. Nesse sentido é possível inferir que os programas de pós-graduação e os grupos de pesquisa possuem uma estreita relação, visto que, nos locais onde existem, há maior consolidação e impulso no trabalho dos grupos, que, em contrapartida, fortalecem a pós-graduação, pois se configuram em espaços que comportam o cerne científico de pesquisadores. Também verificamos que 60,7\% dos grupos estão concentrados na região Sudeste, o que está relacionado com o número de programas de pós-graduação em enfermagem nessa região. Também é interessante observar que aproximadamente 17,64\% pertencem à região Nordeste, 11,76\% estão na região Sul, e apenas 
5,88\% na região Norte, o que demonstra claramente um desequilíbrio em sua distribuição e, consequentemente, da produção científica nacional (Padilha et al., 2012).

Ao compararmos o número de grupos em história da enfermagem com os 410 grupos na área geral de enfermagem registrados na Base Corrente do Conselho Nacional de Desenvolvimento Científico e Tecnológico (CNPq), vimos que representam apenas 8,29\% do total. Mas levando em conta que essa é uma área em expansão e que um número grande de grupos não garante a consolidação da pesquisa nem a sua qualidade, entendemos que a produção dos grupos de pesquisa histórica, em termos de projetos e publicações, é que poderá garantir sua manutenção e seu fortalecimento (Guimarães, Lourenço, Cosac, 2001). Acreditamos que a potencialidade de uma área de conhecimento se manifesta pela qualidade do conhecimento produzido, pelo seu consumo e pela ampliação de estudos nessa área. $\mathrm{O}$ conhecimento produzido pela pesquisa em saúde, se amplamente disseminado, pode ser visto como um bem público global, ao contribuir para o aprimoramento das atividades educativas, assistenciais e políticas, e para a melhoria da saúde individual e populacional (Pang et al., 2003; Noronha et al., 2009).

A relevância deste estudo encontra-se em revelar o cenário da produção acadêmica em história de enfermagem, bem como a evolução de sua linha de pesquisa. Desse modo, este texto tem por objetivo analisar as produções científicas dos grupos de pesquisa em história da enfermagem existentes no Brasil a partir da versão 5.0 do Diretório dos Grupos de Pesquisa no Brasil do CNPq, no período de 1999 a 2009 (Capes, 2010).

\section{Abordagem dos dados}

Trata-se de um estudo exploratório, descritivo, quantitativo do tipo documental (Castiel, Sanz-Valero, 2007), cuja coleta de dados foi realizada no período compreendido entre agosto de 2008 a agosto de 2010. Para a investigação da produção científica dos 34 grupos de pesquisa em história da enfermagem, foram utilizadas duas bases de dados: (a) Diretório dos Grupos de Pesquisa no Brasil do CNPq, versão 5.0, atualizado em março de 2010; (b) currículo individual dos pesquisadores dos grupos de pesquisa disponibilizados na Plataforma Lattes (Currículo Lattes). O Diretório de Pesquisas do CNPq considera como pesquisadores membros graduados ou pós-graduados da equipe de pesquisa, direta e criativamente envolvidos com a realização de projetos e com a produção científica, tecnológica e artística do grupo.

O número total de participantes dos grupos foi de 643, dos quais 161 doutores, 90 mestres, 31 especialistas, 18 técnicos e 343 estudantes: 151 de graduação, 105 de mestrado, 51 de doutorado e 36 de especialização. Do número total de 161 doutores dos grupos, apenas 26 são bolsistas de produtividade do CNPq (12 de nível 1 e 14 de nível 2), que representam 16,14\% do total de doutores. Neste estudo, serão considerados apenas os pesquisadores doutores (135), doutores pesquisadores do CNPq (26), mestres (90), especialistas (31) e graduados (seis), que totalizam então 288 pessoas (Padilha et al., 2012). Na caracterização dos grupos identificamos que 22 grupos pertencem à região Sudeste, seis à região Nordeste, quatro à região Sul e dois à região Norte, totalizando 34 grupos analisados.

A partir dessa definição, passamos a analisar a produção científica de cada pesquisador junto à Plataforma Lattes do CNPq. Realizamos o levantamento da produção relativa a livros, 
capítulos de livros, artigos científicos, trabalhos completos e resumos expandidos. Com relação aos artigos científicos verificamos os veículos de publicação utilizados; a classificação dos periódicos com base nos dados do Qualis Capes (Capes, 2010); período de maior publicação dos grupos; região do pais da qual procedem os autores e qual a proporção de artigos relativos à história da enfermagem. O presente estudo não necessitou ser submetido ao Comitê de Ética da Universidade Federal de Santa Catarina por tratar-se de uma pesquisa documental e ter utilizado apenas fontes de domínio público para sua concretização, porém os pesquisadores seguiram rigorosamente os cuidados éticos na busca, análise e discussão dos resultados.

\section{Resultados e discussão}

Produção científica dos grupos de pesquisa em história da enfermagem no período de 1999 a 2009

No que se refere à análise da produção científica dos grupos de pesquisa em história da enfermagem, a Tabela 1 revela que, do total de 4.784 produções científicas desenvolvidas no período compreendido entre 1999 e 2009, 2.206 (46,1\%) foram artigos científicos; 1.603 $(33,5 \%)$, trabalhos completos e resumos expandidos; 745 (15,5\%), capítulos de livros; e 230 $(4,8 \%)$, livros. Quando analisado o tipo de publicação por grupo, observa-se que $60 \%$ dos grupos seguem o mesmo padrão quantitativo de produção maior de artigos científicos em detrimento das demais formas de publicações. Essa quantidade de artigos publicados vem ao encontro das exigências sofridas especialmente pelos docentes dos programas de pósgraduação stricto sensu em termos de publish or perish. ${ }^{1}$ No Brasil, a realidade não é diferente. Os programas de pós-graduação são avaliados pela qualidade e quantidade de publicações científicas em forma de artigos de seus docentes.

Tabela 1: Produção científica dos grupos de pesquisa em história da enfermagem (1999-2009)

\begin{tabular}{|l|l|}
\hline Tipo de publicação & Quantidade \\
\hline Artigos científicos & 2.206 \\
\hline Trabalhos completos/resumos expandidos & 1.603 \\
\hline Capítulos de livros & 745 \\
\hline Livros & 230 \\
\hline Total & $\mathbf{4 . 7 8 4}$ \\
\hline
\end{tabular}

Fonte: elaborada pelas autoras

O grupo que se destacou com mais produções científicas gerais no período de análise foi o Grupo de Estudos em Saúde Coletiva, vinculado à Universidade Federal do Rio Grande do Sul (UFRGS), pertencente à área da enfermagem, com o total de 481 produções, sendo 201 artigos, 25 livros, 162 capítulos de livros e 93 trabalhos completos e resumos expandidos. A Tabela 1 indica que os artigos científicos representaram quase 50\% de toda a produção científica analisada, considerando-se que, juntamente com livros, capítulos de livros e trabalhos publicados em eventos científicos, constituem as formas mais expressivas de comunicação científica por parte de pesquisadores dos grupos de pesquisa deste estudo.

Esse resultado é corroborado com a análise da produção científica dos grupos de pesquisa em educação em enfermagem da região Sul do Brasil, que apontam a prevalência de $58 \%$ de 
artigos sobre as demais formas de produções científicas (Lino et al., 2010). A produção em ciência e tecnologia tem evoluído com vistas às necessidades concretas, despertadas na prática cotidiana de profissionais de saúde, e, a partir de seus produtos, é capaz de provocar mudanças no processo de trabalho das diferentes estruturas. Nesse âmbito, a atividade científica pode ser mensurada a partir da estatística relativa aos artigos científicos (Erdmann et al., 2005). Nessa perspectiva, entendemos que, na história da enfermagem, a busca pela excelência nas produções científicas é uma constante, fato que podemos observar com o quantitativo de número de artigos produzidos em dez anos pelos grupos de pesquisa em história da enfermagem. A expressividade dessa forma de publicação científica nos remeteu a analisar minuciosamente os 2.206 artigos selecionados no que se refere à publicação anual, à relação com a linha de pesquisa história da enfermagem e aos veículos de publicação.

Ainda que os artigos científicos sejam prevalentes no meio científico deste estudo, é oportuno ressaltar que o grande número de trabalhos completos e resumos expandidos também reflete como ponto positivo a adesão e participação expressiva de docentes, pesquisadores, profissionais e estudantes de graduação e pós-graduação nos eventos da categoria. Nesse contexto, destacamos a importância do incentivo das instituições acadêmicas, dos serviços e dos institutos de pesquisa à participação dos profissionais/docentes/pesquisadores em eventos científicos, no sentido de usufruírem amplamente de tais espaços como meio de socialização e pulverização do conhecimento produzido (Noronha et al., 2009).

Analisando-se o número de artigos publicados no período do estudo, observa-se no Gráfico 1 um perfil de publicação predominantemente progressiva ao longo da década, sendo que, em 2009, o número de artigos publicados dobrou (309 artigos) em comparação ao que foi publicado em 1999 (125 artigos).

Gráfico 1: Publicação anual de artigos pelos grupos de pesquisa em história da enfermagem (1999-2009)

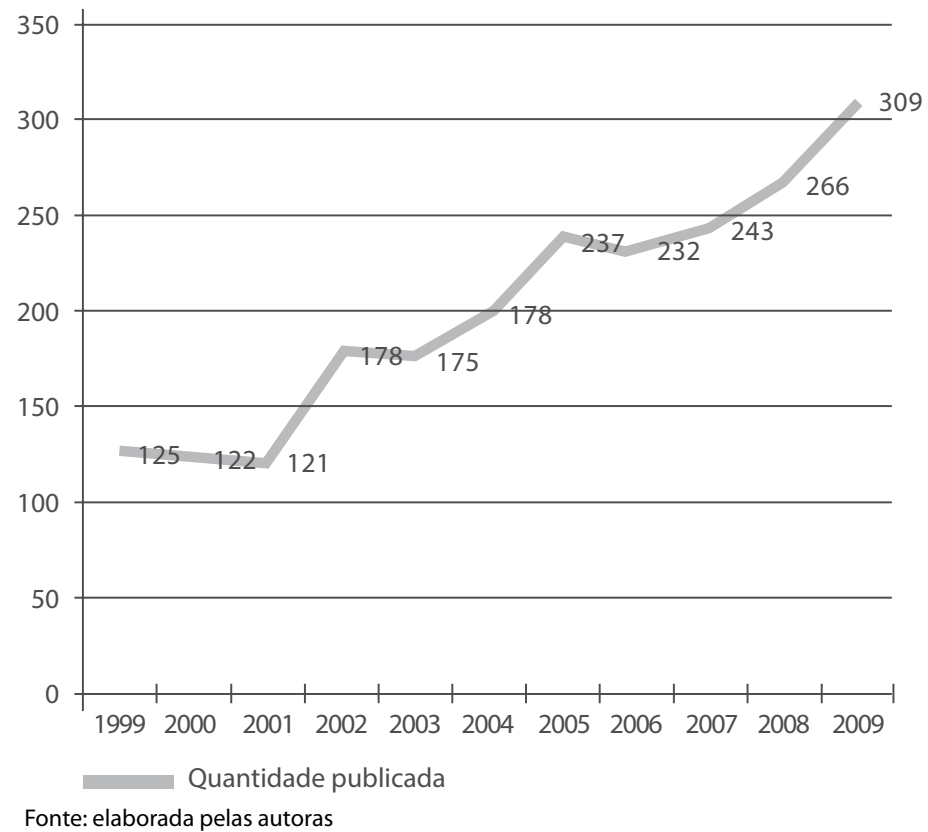


O delineamento da produção de artigos científicos ao longo da década do estudo demonstra um aumento de $247 \%$ de publicações registradas, o que equivale ao dobro da produção anual se utilizados como parâmetros os anos de 1999 e 2009, com respectivamente 125 e 309 artigos científicos. Se pensarmos em número de artigos por pesquisador, o resultado é de 6,5 artigos por ano. Esse resultado mostra-se superior à média apresentada pelo Censo 2008 do CNPq em relação ao número de artigos por ano, por pesquisador, na área da saúde, que é de 2,45 artigos (Brasil, 2011a).

O crescente número de artigos científicos, a nosso ver, decorre do avanço e ampliação dos cursos de pós-graduação sctrictu sensu no país, por diversos motivos. Segundo dados da Capes, o número de cursos de mestrado e doutorado apresentou um avanço de 5,6\% ao ano no período de 1976 a 2004, o que, consequentemente, culminou em 757\% mais mestres e 932\% mais doutores no período de 1987 a 2003 (Brasil, 2011c). É importante lembrar que a formação desses pesquisadores resulta em teses e dissertações as quais geram resultados que são 'fatiados' e que frequentemente são traduzidos para a comunidade acadêmica e científica através de artigos.

O avanço no desenvolvimento científico e tecnológico é fundamental para o crescimento de um país, e, nesse panorama, as pós-graduações da área de enfermagem efetivam-se como propulsoras no desenvolvimento de pesquisadores, em ações investigativas e no protagonismo da construção do conhecimento, acompanhadas e avaliadas continuamente pela Capes (Lino et al., 2010). A partir da década de 1990, com a reestruturação da Capes e com o fortalecimento do enfoque no acompanhamento e na avaliação dos cursos de pós-graduação stricto sensu brasileiros, aprofunda-se o controle de qualidade da comunidade científica e acadêmica, cujo foco tem sido a formação e a qualificação de pesquisadores, destacando-se, nesse sentido, os cursos de doutorado na área de enfermagem, com ênfase na produtividade (Brasil, 2009).

Outra contribuição dos programas de pós-graduação na produção acelerada de artigos científicos é a organização dos pesquisadores em grupos de pesquisa, sendo que $75 \%$ deles surgiram nos últimos dez anos e apresentam dispositivos que facilitam o processo de produção intensiva (Brasil, 2011a; Backes et al., 2009).

Discutem-se, no entanto, possíveis significados da intensa produtividade numérica acadêmica de artigos científicos e sugere-se que o artigo tem assumido papel de mercadoria, como fetiche para legitimar acadêmicos em seus campos de atuação (Castiel, Sanz-Valero, 2007). Em contrapartida, pesquisadores, formuladores de políticas científicas e usuários dos resultados das pesquisas avaliam positivamente o acesso à informação científica e a oportunidade de publicação no país, quando perguntados sobre aspectos relacionados ao ambiente da pesquisa em saúde. Atribui-se essa percepção otimista ao avanço no acesso à informação científica proporcionada por iniciativas nacionais, como o Portal de Periódicos Capes e a Scielo (Scientific eletronic library on-line), e internacionais como a ISI (Web of Science) e Scopus (Noronha et al., 2009; Silva et al., 2009).

A maior visibilidade nacional e internacional da produção científica em enfermagem requer, entre tantos fatores, pesquisadores competentes, ampla política de incentivo à pesquisa, a soma de esforços regionais e determinação no alcance de metas. Desdobra-se, ainda, em dimensão ampliada, tendo em vista que não basta o processo da construção de novos conhecimentos para fortalecer a área de enfermagem, mas o real desafio que se impõe 
aos programas de pós-graduação é que esses produtos concebidos pelos seus pesquisadores sejam consumidos e tenham aplicabilidade na prática profissional, atribuindo benefícios à população a partir de temas de interesse social (Erdmann et al., 2005).

Enfim, a busca do reconhecimento profissional e da valorização de cada área de conhecimento é um desafio enfrentado pela área de história da enfermagem e que pode ser concretizada à medida que amplia a sua visibilidade por meio da produção científica, realização de eventos específicos e da ampliação de núcleos e grupos em todo o território nacional. Vale ressaltar também que o campo da história da enfermagem vem se beneficiando das investigações realizadas por historiadores, sociólogos, psicólogos, entre outros, acerca das profissões de saúde, e entre elas, a enfermagem, contribuindo com um olhar diferenciado para a compreensão da profissão.

\section{Onde se publica e qual o impacto da produção dos artigos científicos}

A produção científica tem o intuito de formar um corpo de conhecimento próprio de uma disciplina, desenvolver novas tecnologias e atender as exigências da sociedade. Nesse contexto, as revistas especializadas são importantes veículos de comunicação da produção científica, pois possibilitam a divulgação do processo e do produto dessa atividade (Umbelino, 2008).

Os 2.206 artigos produzidos pelos pesquisadores dos grupos de pesquisa em história da enfermagem, entre os anos de 1999 e 2009, foram publicados em 297 revistas. Desse total, $68 \%$ foram publicados em revistas de enfermagem (1.499 artigos) e 32\% em revistas de outras áreas do conhecimento (707), quais sejam: 4,9\% (medicina), 6,2\% (psicologia), 6,2\% (área interdisciplinar), 3,7\% (saúde coletiva), 2,2\% (educação), 1,8\% (ciências humanas e sociais), 0,5\% (odontologia, fisioterapia e nutrição) e 6,5\% em periódicos de áreas diversas. Analisando essa produção, podemos perceber que há um esforço em fomentar o corpo de conhecimento próprio da enfermagem enquanto ciência e disciplina. Para uma profissão ser reconhecida no âmbito nacional e internacional e ter visibilidade no cenário científico é necessária a produção de sua própria ciência. Isso se traduz no volume da produção publicada em revistas científicas da área específica ou das áreas afins (Cunha, Marques, 2007).

Nesse sentido, a enfermagem, além de produzir e divulgar seu conhecimento em revistas da sua própria área, tem uma produção significativa que dialoga com outras áreas do conhecimento, tanto no âmbito das ciências da saúde como no de outras ciências. Isso pode ser observado pelo volume de publicações em periódicos não exclusivos da enfermagem. Esses dados corroboram com as tendências da pesquisa em saúde, que extrapolam progressivamente as fronteiras tradicionais das ciências da saúde, incorporando conhecimentos de outras áreas.

Um recurso de análise importante sobre qualidade e excelência acadêmica é a qualidade da produção científica, mensurada pela classificação do sistema Qualis Periódicos. O modelo foi criado pela Capes em 1998, visando classificar os periódicos científicos usados na divulgação da produção intelectual dos programas de mestrado e doutorado do país. Essa classificação divide atualmente os periódicos em oito estratos, A1, A2, B1, B2, B3, B4, B5 e C, correspondendo A1 ao maior peso (100) e C ao menor peso (0). Essa estratificação é derivada da análise de parâmetros formais de avaliação de periódicos científicos, como formato, existência de número de registro no International Standard Serial Number (ISSN), periodicidade, conteúdo científico representativo, corpo editorial com qualificação, revisão dos artigos por pares, 
seguimento das normas da Organização Mundial dos Editores Científicos (Wame), inserção dos periódicos em bases de dados e outros índices bibliométricos, tais como o fator de impacto do ISI (Web of Science) e o índice H no SCImago, como indicadores dos melhores periódicos classificados para todas as áreas do conhecimento científico. Na área de enfermagem, além dos indicadores citados, é relevante também o índice bibliométrico RIC, da base indexadora Cuiden, por aglutinar 447 revistas científicas indexadas, entre elas 252 de enfermagem, marcadas por uma produção ibero-americana, mais próxima da realidade brasileira (Capes, 2010; Brasil, 2011b; Ruiz, Greco, Braile, 2009; Erdmann et al., 2009). Em relação à produção de artigos pelos pesquisadores dos grupos analisados, apresentamos o Quadro 1, com os dez periódicos com maior frequência de publicações:

Quadro 1: Periódicos com maior número de publicações dos grupos de pesquisa de história de enfermagem 1999-2009

\begin{tabular}{|l|l|l|}
\hline Periódicos & Estrato & Total \\
\hline Revista Brasileira de Enfermagem & B1 & 230 \\
\hline Escola Anna Nery - Revista de Enfermagem & B1 & 143 \\
\hline Revista da Escola de Enfermagem da USP & A2 & 127 \\
\hline Texto e Contexto Enfermagem & A2 & 98 \\
\hline Ciência, Cuidado e Saúde & B2 & 95 \\
\hline Revista Brasileira de Crescimento e Desenvolvimento Humano & B3 & 89 \\
\hline Revista Latino-americana de Enfermagem & A2 & 84 \\
\hline Acta Paulista de Enfermagem & A2 & 69 \\
\hline Revista Enfermagem UERJ & B1 & 65 \\
\hline Online Brazilian Journal of Nursing & B1 & 55 \\
\hline
\end{tabular}

Fonte: elaborado pelas autoras

Quanto ao total da produção dos artigos (2.206), dos dez periódicos com mais publicações, a Revista Brasileira de Enfermagem (Reben) obteve o maior volume, com 10,4\% do total. Criada em 1932 para estabelecer a interlocução com os associados da Associação Brasileira de Enfermagem (Aben), a Reben representa nacionalmente um marco para a compreensão da enfermagem enquanto profissão, com papel fundamental em sua trajetória histórica. Apesar de assemelhar-se a outros periódicos com relação a termos de normalização, periodicidade e tipos de artigos publicados, a Reben mantém a característica marcante de representatividade (Mancia, 2007). Nesse sentido, apesar de não estar ligada diretamente a uma instituição acadêmica, mantém alto teor científico, além de grande visibilidade nacional, contribuindo para a consolidação profissional da enfermagem em nosso país.

Vale destacar também que o sexto periódico com maior número de publicações foi a Revista Brasileira de Crescimento e Desenvolvimento Humano, que é uma revista interdisciplinar vinculada à área da psicologia, mantendo uma significativa produção dos pesquisadores dos grupos estudados (4,03\%). Dos artigos publicados pelos pesquisadores dos grupos de história da enfermagem, 79,4\% estavam em periódicos classificados pelo sistema Capes como área de enfermagem, 14,1\% em periódicos da área interdisciplinar, saúde coletiva, medicina, história, letras/linguística, e 6,5\% em periódicos ainda não cadastrados no sistema Capes Qualis. Quanto à proporção do estrato de classificação de periódicos pelo Qualis, apenas $0,4 \%$ pertencia ao estrato $\mathrm{A} 1$, nenhum deles sendo revista de enfermagem. Dos demais, 
$19,5 \%$ pertenciam ao estrato $\mathrm{A} 2 ; 27,1 \%$ ao estrato $\mathrm{B} 1 ; 18,2 \%$ ao estrato $\mathrm{B} 2 ; 12,7 \%$ ao estrato $\mathrm{B} 3 ; 7,9 \%$ ao estrato $\mathrm{B} 4 ; 6,3 \%$ ao estrato $\mathrm{B} 5$; e $1,3 \%$ ao estrato $\mathrm{C}$, entre os quais não havia periódico de enfermagem.

Desse modo, observamos que os grupos de trabalho analisados publicam com maior frequência em periódicos pertencentes ao estrato B1. É possível notar um deficit importante de publicações em revistas com classificação A1, demonstrando dificuldade em internacionalizar a produção científica da área, o que se soma à ausência de periódicos de enfermagem brasileiros que tenham essa classificação, bem como à dificuldade de os autores publicarem em revistas internacionais, contribuindo para baixa visibilidade internacional da enfermagem brasileira no que diz respeito a sua produção científica (Marziale, 2005).

\section{As temáticas dos artigos científicos sobre história da enfermagem - uma análise prospectiva}

A pesquisa em enfermagem é uma forma de conhecimento da realidade e possível intervenção objetiva nos processos de trabalho em saúde e nos processos de formação e educação, entre outros (Egry, Fonseca, 2006). É por meio da pesquisa que poderá ser alcançado o crescimento e o desenvolvimento da área de história da enfermagem, que é essencial para a compreensão e visibilidade enquanto parte da ciência da enfermagem (D'Antonio, 2009). A reserva de espaço no mercado de trabalho e na produção do conhecimento em história da saúde e enfermagem ainda está em processo de consolidação. Ela se dá por meio da atuação dos grupos de pesquisa em história da enfermagem, bem como do desenvolvimento de sua produção técnico-científica em articulação com o ensino da enfermagem nos programas de graduação e pós-graduação. Esses são propulsores importantes capazes de promover uma consciência segura e fortalecida da enfermagem como ciência e profissão.

Ao analisar os artigos científicos dos pesquisadores dos grupos de pesquisa em história da enfermagem, entendemos que seria importante avaliar o quanto dessa produção se referia mais especificamente à temática 'história da enfermagem', vistos sua relevância específica para a área e o impacto de suas publicações. Logo, procedeu-se à análise dos 2.206 artigos destacados neste estudo e identificaram-se, pelos títulos, quais apresentavam abordagem relativa à história da enfermagem. Constatou-se que 394 artigos possuem efetivamente relação com a linha de pesquisa histórica, enquanto os demais (1.808) abordam outras temáticas inerentes aos interesses da área da enfermagem em geral. A análise dos 394 artigos publicados indica que a produção sobre história da enfermagem representa 18,04\% da produção total dos grupos de pesquisa em enfermagem no Brasil, o que significa um vasto material bibliográfico sobre o assunto e nos permite conhecer e analisar a evolução dessa profissão, elucidando o contexto vivido e fornecendo os significados desse contexto.

Os artigos de história da enfermagem analisados (394) foram organizados em categorias segundo estudo realizado anteriormente (Padilha et al., 2007), que identificou cinco grandes áreas de produção de conhecimento em história da saúde e enfermagem: identidade profissional da enfermagem; institucionalização da enfermagem; escolas de enfermagem; entidades organizativas; e especialidades de enfermagem. Utilizamos então a mesma classificação para analisar os 394 artigos científicos encontrados nos currículos dos pesquisadores e identificados como tal pelos títulos e/ou resumos (Tabela 2). Nesse estudo, não foram consultados os artigos na íntegra. 
Tabela 2: Categorização dos artigos de história da enfermagem produzidos pelos grupos de pesquisa em enfermagem, 1999-2009

\begin{tabular}{|l|l|}
\hline Caracterização dos artigos & Total \\
\hline Identidade profissional da enfermagem & 112 \\
\hline Institucionalização da enfermagem & 25 \\
\hline Escolas de enfermagem & 105 \\
\hline Entidades organizativas de enfermagem & 30 \\
\hline Especialidades de enfermagem & 101 \\
\hline Outros & 21 \\
\hline Total & 394 \\
\hline
\end{tabular}

Fonte: elaborada pelas autoras

Os resultados quantitativos encontrados na categorização dos estudos seguem uma lógica aproximadamente semelhante àquela encontrada na pesquisa realizada com teses e dissertações disponíveis no Centro de Estudos e Pesquisa em Enfermagem (Cepen) da Associação Brasileira de Enfermagem (Aben). O que indica, de certo modo, que a produção científica em artigos tem uma aderência constante às teses e dissertações defendidas nos diversos programas de pós-graduação em enfermagem ou outras áreas. A inclusão dos artigos em cada uma das categorias antes mencionadas não pretende ser definitiva, mas apenas possibilitar um olhar sobre o que está sendo produzido ao longo do tempo acerca da temática central deste estudo.

A maior parte dos artigos relativos à história da enfermagem e saúde publicados pelos pesquisadores tem como foco central a identidade profissional da enfermagem (112). Em relação ao que constituímos como identidade profissional de enfermagem se incluem os artigos relativos a: biografias, questões de gênero, imagem profissional tanto positiva quanto negativa, emblemas, uniformes e o cuidar enquanto condição de identidade profissional. Esse resultado demonstra uma preocupação dos pesquisadores com a identidade da profissão e consequente clarificação dos papéis femininos e masculinos exercidos nos espaços profissionais (Padilha, Nelson, Borenstein, 2011).

A segunda categoria com o maior número de artigos publicados foi a das escolas de enfermagem (105). Nesse item incluímos todos os artigos que tratavam de aspectos relativos à criação de escolas de enfermagem no Brasil ao longo do século XX, e também de ensino, pesquisa, estruturas curriculares, assim como, da história de programas de pós-graduação em enfermagem. Percebe-se o interesse dos pesquisadores com a inserção dos conteúdos relativos à história da enfermagem nos currículos dos cursos de graduação e também de pósgraduação brasileiros, articulando-se a uma preocupação mundial com o assunto e referida pela Associação Americana de História da Enfermagem (AAHN).

Outra categoria de grande destaque em termos de artigos científicos é a relativa às especialidades na enfermagem (101). Nela encontramos artigos sobre a criação das especialidades em enfermagem brasileiras, especialmente nas áreas de saúde da mulher, da criança, administração e saúde pública. Essa categoria tem uma aderência direta com o que encontramos sobre a institucionalização da enfermagem, considerando que, ao tratar do modo como a enfermagem foi se institucionalizando, também apresenta o tipo e a característica de cada uma das instituições. Em institucionalização da enfermagem (25), incluímos os artigos que tratavam da criação de hospitais e da inclusão da enfermagem e das enfermeiras nos campos de prática hospitalares e de saúde pública. 
Quanto à área definida como entidades organizativas da enfermagem (30), pensamos que ainda precisa avançar em termos de produção científica, pois tanto em relação a teses e dissertações quanto em relação a artigos o número é bem pouco significativo. A maioria deles trata de aspectos da Associação Brasileira de Enfermagem e do Movimento Participação, iniciado na década de 1980. A categoria outros, criada para ressaltar a produção de conhecimento acerca dos aspectos teórico-metodológicos da história da enfermagem (21), constitui uma literatura importante e instrumentalizadora de estudos com perspectiva histórica.

Sabemos que desenvolver a pesquisa histórica tendo em vista a construção da memória da enfermagem e analisar criticamente a história das enfermeiras e da enfermagem é um desafio a ser enfrentado crescentemente. Para tanto, o registro sistematizado da história da enfermagem nas diversas faces e fases desenvolvidas nas diferentes regiões do mundo poderá ser um exercício de autoconhecimento dos enfermeiros com consequente explicação de sua identidade enquanto profissão (Nelson, 2009). Desse modo, o reconhecimento por parte da sociedade possivelmente virá por meio de uma prática exercida efetivamente e consubstanciada pelo próprio desempenho de profissionais da enfermagem, sendo que isso ainda é outro desafio a ser enfrentado.

Embora a análise tenha sido realizada a partir dos grupos de pesquisa que se classificaram da área de história da enfermagem (de acordo com a busca no diretório CNPq), compreendemos que, dentro de um grupo, existem diversos eixos temáticos e, geralmente, um eixo central. A maioria dos grupos trata do tema da história da enfermagem como um eixo secundário. A história da enfermagem quase sempre está associada a outros grupos, apresentando-se como uma das linhas de pesquisa e não como foco principal, enquanto em outras áreas do conhecimento, como saúde da mulher e saúde da criança, existe maior quantidade de grupos específicos que estudam seu tema principal.

\section{Considerações finais}

A investigação acerca da produção de conhecimento pelos 34 grupos de pesquisa em história da enfermagem existentes no Brasil complementa qualitativamente o estudo anterior relativo à caracterização desses grupos na realidade brasileira (Padilha et al., 2012) . Os resultados nos permitem inferir que a produção científica desses grupos vem evoluindo significativamente ao longo dos anos e confirmam a sua articulação com os cursos de graduação e pós-graduação em enfermagem.

Vale ressaltar a preocupação dos pesquisadores com relação à identidade profissional da enfermagem. Os estudos atuais vêm permitindo a análise das relações de gênero no terreno do trabalho privado/familiar e no da entrada da mulher no mundo do trabalho público/ profissional. Também permitem aos pesquisadores relativizar leituras maniqueístas acerca das posições de gênero. Tais posições tendem a encobrir a complexidade que permeia as relações sociais e as produções dos sujeitos no cotidiano das práticas sociais. Por exemplo, o autoritarismo sempre referido nas relações entre médicos e enfermeiras no cotidiano hospitalar tende, por vezes, a encobrir as relações autoritárias que são exercidas entre as enfermeiras e os demais membros da equipe de enfermagem, ou até mesmo na relação de cuidado enfermagem/paciente. 
Embora a produção científica na sua totalidade não assegure a vinculação com estudos de perspectiva histórica, indica possivelmente a participação dos pesquisadores em mais de uma linha de pesquisa além da história da enfermagem propriamente dita. O expressivo número de artigos, trabalhos completos/resumos, capítulos de livros e livros reflete positivamente a participação de docentes, discentes e profissionais nos grupos de pesquisa em história da enfermagem. Porém, um fator limitador do estudo foi os artigos terem sido incluídos na área de história da enfermagem e saúde somente pela leitura dos seus títulos e não do seu conteúdo completo. Conclui-se que a divulgação e socialização dos conhecimentos em história da enfermagem nos últimos dez anos demonstram que essa área já se encontra consolidada, mesmo que o total da produção de artigos não seja diretamente relacionada à história da enfermagem, mas, sim, às linhas de pesquisa dos investigadores e valorização dos grupos de pesquisa nesta área.

\section{AGRADECIMENTOS}

Agradecemos o apoio do Conselho Nacional de Desenvolvimento Científico e Tecnológico (CNPq) para o desenvolvimento desta pesquisa.

\section{NOTAS}

* O presente trabalho é produto do Grupo de Estudos da História do Conhecimento da Enfermagem e Saúde (GEHCES), grupo de pesquisa do CNPq, do qual Maria Itayra Padilha é líder e as demais autoras são membros.

${ }^{1}$ A máxima 'publicar ou morrer' foi cunhada em 1932 por Harold Jefferson Coolidge e Robert Howard Lord em seu livro Archibald Cary Coolidge: life and letters. Posteriormente, espalhou-se pelo mundo com o mesmo significado, ou seja, publicar a qualquer custo para manter-se ativo no mercado científico e acadêmico.

\section{REFERÊNCIAS}

BACKES, Vânia Marli Schubert et al.

Grupos de pesquisa de educação em enfermagem da região sul do Brasil. Revista Gaúcha Enfermagem, Porto Alegre, v.30, n.2, p.249-56. 2009.

BRASIL.

Ministério da Ciência e Tecnologia. Conselho Nacional de Desenvolvimento Científico e Tecnológico - CNPq. Diretório dos Grupos de Pesquisa, Séries Históricas [base de dados online]. Disponível em: http://dgp.cnpq.br/censos/series_ historicas/index_basicas.htm. Acesso em: 9 maio 2011. 2011a.

BRASIL.

Ministério da Educação. Coordenação de Aperfeiçoamento de Pessoal de Nível Superior. Qualis/Capes. Disponível em: http://qualis. capes.gov.br/webqualis/ConsultaPeriodicos.faces. Acesso em: 28 jun. 2011. 2011b.

BRASIL.

Ministério da Educação. Estatísticas [internet]. Disponível em: http://www.capes.gov.br/ estatisticas. Acesso em: 3 maio 2011. 2011c.
BRASIL.

Ministério da Educação. Coordenação de Aperfeiçoamento de Pessoal de Nível Superior. Capes: história e missão. Disponível em: http:// www.capes.gov.br/sobre-a-capes/historia-emissao. Acesso em: 16 fev. 2009. 2009.

CAPES.

Coordenação de Aperfeiçoamento de Pessoal de Nível Superior. Relatório de avaliação 20072009: trienal 2010. Disponível em: http://trienal. capes.gov.br/wp-content/uploads/2010/09/ ENFERMAGEM-rel-11set10.pdf. Acesso em: 27 jun. 2001. 2010.

CASTIEL, Luis David; SANZ-VALERO, Javier. Entre fetichismo e sobrevivência: o artigo científico é uma mercadoria acadêmica? Cadernos de Saúde Pública, Rio de Janeiro, v.23, n.12, p.3041-3050. 2007.

CUNHA, Isabel Cristina Kowal Olm; MARQUES, Isaac Rosa.

Socializando a produção científica da enfermagem do Piauí. Revista Brasileira de Enfermagem, Brasília, v.60, n.4, p.369-369. 2007. 
D'ANTONIO, Patricia.

Thinking about place: researching and reading the global history of nursing. Texto e Contexto Enfermagem, Florianópolis, v.18, n.4, p.766-772. 2009.

EGRY, Emiko Yoshikawa; FONSECA, Rosa Maria Godoy Serpa da.

Pesquisa em enfermagem: por uma pedagogia da ética! Revista da Escola de Enfermagem da USP, São Paulo, v.40, n.2, p.148-150. 2006.

ERDMANN, Alacoque Lorenzini et al. A avaliação de periódicos científicos Qualis e a produção brasileira de artigos da área de enfermagem. Revista Latino-Americana de Enfermagem, Ribeirão Preto, v.17, n.3, p.403-409. 2009.

ERDMANN, Alacoque Lorenzini et al. Teses produzidas nos programas de Pósgraduação em Enfermagem de 1983 a 2001. Revista da Escola de Enfermagem da USP, São Paulo,v.39, n.especial, p.497-505. 2005.

GUIMARÃES, Reinaldo; LOURENÇO, Ricardo; COSAC, Silvana.

A pesquisa em epidemiologia no Brasil. Revista de Saúde Pública, São Paulo, v.35, n.4, p.321-40. 2001.

LINO, Mônica Motta et al.

Perfil da produção científica e tecnológica dos grupos de pesquisa em educação em enfermagem da região sul do Brasil. Revista Latino-Americana de Enfermagem, Ribeirão Preto, v.18, n.3, p.452-458. 2010.

MANCIA, Joel Rolim.

Revista Brasileira de Enfermagem e seu papel na consolidação profissional. Tese (Doutorado) Programa de Pós-graduação em Enfermagem, Universidade Federal de Santa Catarina, Florianópolis. 2007.

MARZIALE, Maria Helena Palucci. Produção científica da enfermagem brasileira: a busca pelo impacto internacional. Revista LatinoAmericana de Enfermagem, Ribeirão Preto, v.13, n.3, p.285-286. 2005.

NELSON, Sioban.

Historical amnesia and its consequences: the need to build histories of practice. Texto $e$ Contexto - Enfermagem, Florianópolis, v.18, n.4, p.781-787. 2009.
NORONHA, José et al.

Análise do sistema de pesquisa em saúde do Brasil: o ambiente de pesquisa. Revista Saúde Sociedade, São Paulo, v.18, n.3. 2009.

PADILHA, Maria Itayra et al. Grupos de pesquisa em história da enfermagem: a realidade brasileira. Revista da Escola de Enfermagem da USP, São Paulo, v.46, n.1, p.192-199. 2012.

PADILHA, Maria Itayra et al.

A produção da pesquisa histórica vinculada aos programas de pós-graduação no Brasil, 1972 a 2004. Texto e Contexto - Enfermagem, Florianópolis, v.16, n.4, p.671-679. 2007.

PADILHA, Maria Itayra; NELSON, Sioban; BORENSTEIN, Miriam Susskind.

As biografias como um dos caminhos na construção da identidade do profissional da enfermagem. História, Ciências, SaúdeManguinhos, Rio de Janeiro, v.18, supl.1, p.241-252. 2011.

PANG, Tikki et al.

Knowledge for better health: a conceptual framework and foundation for health research systems. Bulletin of the World Health Organization, Washington, v.81, n.11, p.815-20. 2003.

RODRIGUES, Rosalina Partezani et al.

A formação de doutores em enfermagem no Brasil. Texto e Contexto - Enfermagem, Florianópolis, v.11, n.3, p.66-76. 2002.

RUIZ, Milton Artur; GRECO, Oswaldo Tadeu; BRAILE, Domingo Marcolino.

Fator de impacto: importância e influência no meio editorial, acadêmico e científico. Revista Brasileira de Cirurgia Cardiovascular, São José do Rio Preto, v.24, n.3, p.273-278. 2009.

SILVA, Maria Júlia Paes da et al.

Produção do conhecimento em enfermagem: da ideia da pesquisa à publicação em periódico qualificado. Revista da Escola de Enfermagem da USP, São Paulo, v.43, n.esp.2, p.1347-1351. 2009.

UMBELINO, Fernanda Maria.

Factor de impacto de revistas científicas na área de enfermagem. Referência, Coimbra, série 2, n.8, p.95-100. 2008.

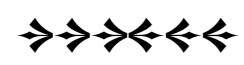




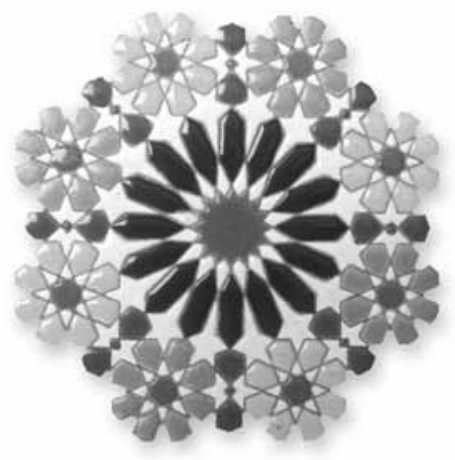

